\title{
3-D imaging of particle tracks in solid state nuclear track detectors
}

\author{
D. Wertheim ${ }^{1}$, G. Gillmore ${ }^{2}$, L. Brown ${ }^{3}$, and N. Petford ${ }^{4}$ \\ ${ }^{1}$ Faculty of Computing IS \& Maths, Kingston University, Surrey, KT1 2EE, UK \\ ${ }^{2}$ Faculty of Science, Kingston University, Surrey, KT1 2EE, UK \\ ${ }^{3}$ Advanced Engineered Materials Group, National Physical Laboratory, Teddington, Middlesex, UK \\ ${ }^{4}$ Bournemouth University, Dorset, UK
}

Received: 27 November 2009 - Revised: 7 April 2010 - Accepted: 15 April 2010 - Published: 20 May 2010

\begin{abstract}
It has been suggested that 3 to $5 \%$ of total lung cancer deaths in the UK may be associated with elevated radon concentration. Radon gas levels can be assessed using CR-39 plastic detectors which are often assessed by 2-D image analysis of surface images. 3-D analysis has the potential to provide information relating to the angle at which alpha particles impinge on the detector. In this study we used a "LEXT" OLS3100 confocal laser scanning microscope (Olympus Corporation, Tokyo, Japan) to image tracks on five CR-39 detectors. We were able to identify several patterns of single and coalescing tracks from 3-D visualisation. Thus this method may provide a means of detailed 3-D analysis of Solid State Nuclear Track Detectors.
\end{abstract}

\section{Introduction}

Inhalation of radon gas $\left({ }^{222} \mathrm{Rn}\right)$ and associated ionising decay products is known to be a cause of lung cancer in humans (Darby et al., 2005; ICRP, 2007). It has been suggested that 3 to $5 \%$ of total lung cancer deaths in the UK can be associated with elevated radon concentrations in the home and/or workplace. Thus radon monitoring in buildings is routinely undertaken in areas of known risk. Some organisations, for example the Radon Council in the UK and the Environmental Protection Agency in the USA, advocate a "to test is best" policy (Papworth, 1997). Radon gas occurs naturally, arising from decay of ${ }^{238} \mathrm{U}$ in rock and soils.

Assessment of radon gas concentration can be made with CR-39 plastic detectors (Phillips et al., 2004) which conventionally are assessed by 2-D image analysis of surface images; however there can be some variation even in closely spaced detectors (Cliff and Gillmore, 2001). Several radon

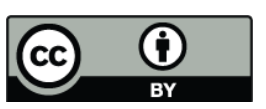

Correspondence to: D. Wertheim (d.wertheim@kingston.ac.uk) measurement methods are used such as activated carbon and electrets; the most widely used measurement method is CR-39 solid state nuclear track-etch detectors (SSNTDs). In this technique, heavily ionising alpha particles form tracks as a result of radiation damage to the detector from interaction between alpha particles and the molecules making up the CR-39 polymer.

We have previously described confocal microscopy methods to obtain 3-D data for particle track visualisation and analysis (Petford et al., 2005; Wertheim et al., 2009). Although 3-D imaging of the tracks could be more timeconsuming than 2-D analysis, 3-D analysis has the potential to provide information relating to angle at which the alpha particle hits the detector as well as possibly indicating the energy of alpha particles. The aim of this study was to investigate the 3-D appearance of isolated single and coalescing multiple tracks in SSNTDs from confocal microscope images.

\section{Method}

A "LEXT" OLS3100 confocal laser scanning microscope (Olympus Corporation, Tokyo, Japan) was used in confocal mode to obtain 3-D image data on five CR-39 plastic detectors as previously described (Wertheim et al., 2009); the detectors were from the Radon Metrology Laboratory at Kingston University. In summary the detectors were placed on a glass slide which was then positioned on the microscope stage. Confocal images were obtained using a $\times 50$ or $\times 100$ objective; both lenses having a Numerical Aperture (NA) of 0.95 . The upper and lower levels required for the $\mathrm{z}$ direction were assessed manually in order to image the full depth of the tracks. Using the $\times 100$ objective the microscope system has a planar resolution of up to $0.12 \mu \mathrm{m}$ and height resolution up to $0.01 \mu \mathrm{m}$.

Published by Copernicus Publications on behalf of the European Geosciences Union. 
(a)

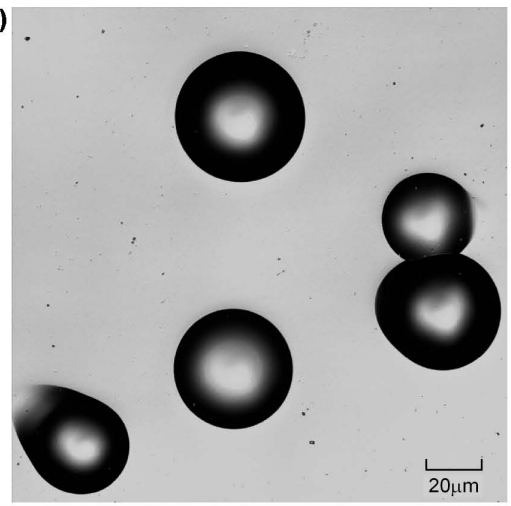

(c)

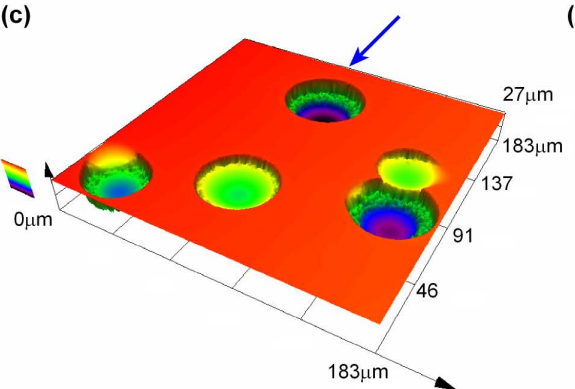

(b)

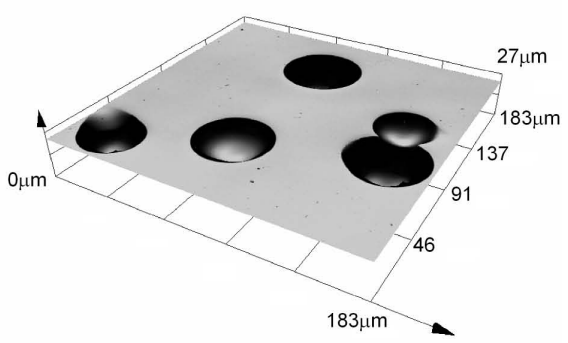

(d)

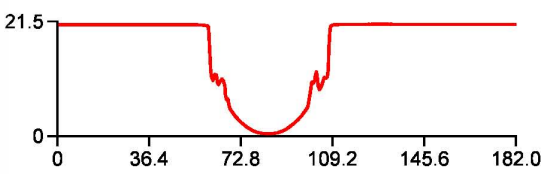

Fig. 1. Example of 3-D visualisation of tracks from data obtained with a $\times 50$ objective showing the distribution of single isolated tracks and two coalescing tracks. (a) shows the view from the top surface, (b) shows a side view and (c) the corresponding height encoded colour image with red being the top, through yellow, blue, green to purple being the deepest. (d) shows depth profile (in $\mu \mathrm{m}$ ) for the deepest track (blue arrow in c) which has a measured depth of $20 \mu \mathrm{m}$.

Initial microscope examination was made with $\mathrm{a} \times 10 \mathrm{ob}-$ jective lens in order to identify regions of the detector with single and coalescing multiple tracks. Regions were then examined on each SSNTD using confocal microscopy and the resultant 3-D images visually assessed.

\section{Results}

Examination of the five detectors showed different types of track appearance. A number of patterns of single isolated and multiple coalescing track formation patterns were identified as shown in the following examples. In addition tracks were identified with different angles and depths with respect to the detector surface.

The example in Fig. 1 shows three single and a double track consisting of two apparently different tracks. The lower left single track indicates the particle impinged the detector at an acute angle (with respect to the detector surface) as the diameter of the upper left aspect is clearly lower than in the centre.

Figure 1a and $\mathrm{b}$ shows grey-scale views from the top surface (a) and side (b). Figure 1c shows a colour coded 3-D visualisation of the tracks which helps to confirm the appearance of the lower left single track. The colour visualisation also suggests that the double tracks were formed by two separate particles as the two tracks are overlapping, of very dif- ferent depths and the strike angles do not suggest a bouncing particle. The near symmetrical profile in Fig. 1d suggests that the top hit was formed by a particle approaching the detector at right angles to the surface.

Figure 2 also shows three isolated single tracks and a double track. In this case the grey-scale images clearly show that the coalescing double tracks are likely to be formed from two separate particles hitting the detector since the tracks are inclined in different directions. The colour coded height image confirms the inclination angles of the two coalescing tracks and helps to show the variation in depth. The maximum depth of $23 \mu \mathrm{m}$ was seen in a single isolated track (top left). A depth profile trace (in $\mu \mathrm{m}$ ) of the right track from the two coalescing tracks is shown in Fig. 2 d.

An example of a triple hit region is shown in Fig. 3. In the example there is no clear variation in the strike angle of the three tracks.

Figure 4 shows that track size can vary as well as the angle of the tracks on the detector. The elliptical shaped track on the lower left of the image appears to have hit the detector at a higher angle with respect to the normal to the track detector when compared with the upper right track which appears circular.

The number of clear single and coalescing tracks was assessed from a 2-D image of one detector obtained using a $\times 10$ objective. In a region size of $1.3 \mathrm{~mm}$ by $1.0 \mathrm{~mm}$, there 
(a)

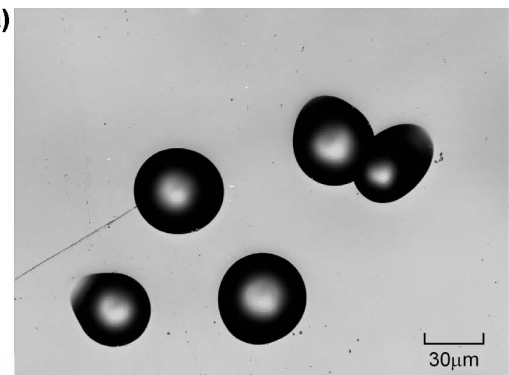

(c)

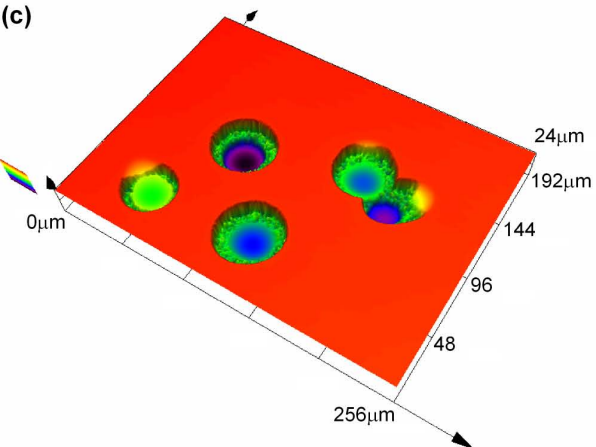

(b)

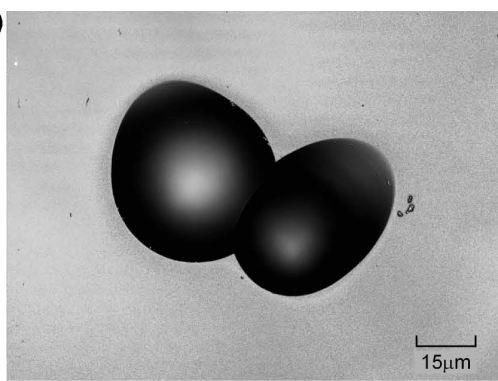

(d)

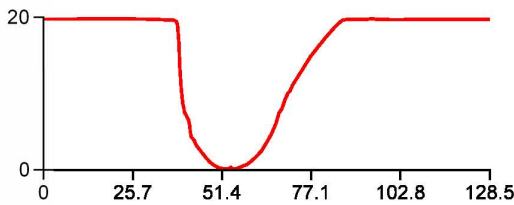

Fig. 2. Example of 3-D visualisation of tracks from data showing the distribution of single isolated tracks and two coalescing tracks. (a) shows the view from the top surface obtained with a $\times 50$ objective and (b) shows a close up image of the two coalescing tracks obtained with a $\times 100$ objective. (c) shows a side view height encoded colour image with red being the top, through yellow, blue, green to purple being the deepest. (d) shows the height profile variation for the right track forming the two coalescing tracks. The profile is from left to right through the track's long axis and shows a steeper slope on the left hand side compared with the right hand side suggesting that the particle hit the detector from the right.

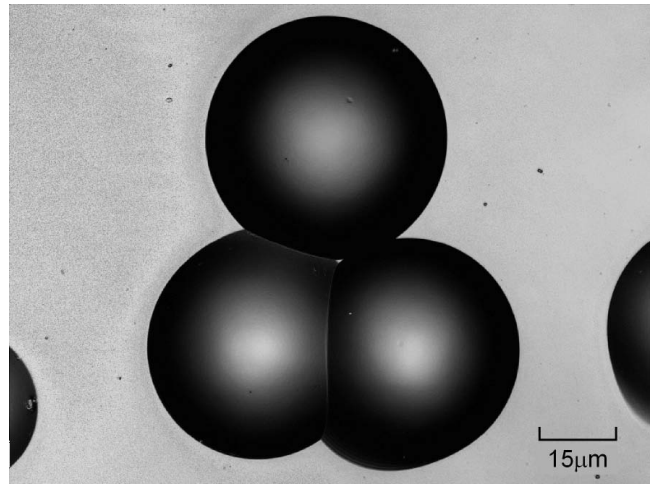

Fig. 3. Example using a $\times 100$ objective of three coalescing tracks.

were 51 clear single tracks, 8 double tracks (i.e. apparently consisting of two coalescing tracks) and 2 triple tracks (i.e. apparently consisting of three coalescing tracks); in this assessment tracks on the border of the image were not included.

The examples show that there are a variety of track patterns both in terms of individual track shape and the proximity between tracks. Furthermore it can be seen that the depth profile not only provides the height of the track but also should help to infer the likely angle at which the particle may have hit the detector.

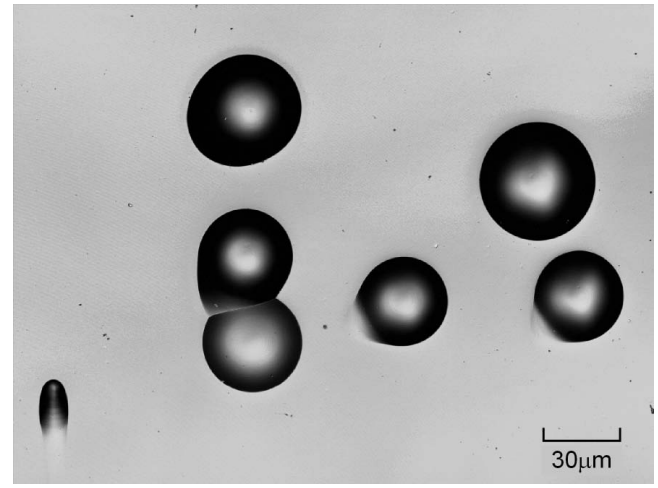

Fig. 4. Example showing variation in track diameter. Image obtained using a $\times 50$ objective.

\section{Conclusions}

From the assessment of the number of clear single and coalescing tracks on the 2-D image of one detector, 22 tracks were part of coalescing tracks ( 16 tracks were part of the 8 coalescing double tracks and 6 part of the two coalescing triple tracks); hence $30 \%$ (22 of 73 ) of the observed tracks were coalescing, highlighting the importance of identifying such tracks. 
In this study we have successfully used high resolution confocal microscopy to investigate tracks in five SSNTDs. We have observed different patterns of track formation for example single isolated tracks and multiple coalescing tracks. These observations suggest that it may be difficult to interpret the appearance of coalescing tracks and distinction from artefact from only 2-D analysis. Furthermore the 3-D confocal microscopy technique enables identification and quantification of track angle, area and depth and may help in objective characterisation of tracks.

The angle of particle strike is likely to be an indicator of alpha particle direction. Together with area and depth data this may help to give a comprehensive assessment of tracks. Thus 3-D visualisation and image analysis can enable characterisation of track features; hence this method may provide a means of rapid and detailed 3-D analysis of SSNTDs which could help to improve assessment accuracy.

Acknowledgements. We are grateful to Claire Ivison for help in preparing the figures. The images in this paper are Crown Copyright.

Edited by: R. Crockett

Reviewed by: two anonymous referees

\section{References}

Cliff, K.and Gillmore, G. K. (Eds.): The Radon Manual: A Guide to the Requirements for the Detection and Measurement of Natural Radon Levels, 3rd edn., Associated Remedial Measures and Subsequent Monitoring of Results, The Radon Council, Shepperton, Middlesex, 2001.

Darby, S., Hill, D., Auvinen, A., Borros-Dios, J. M., Baysson, H., Bochicchio, F., Deo, H., Falk, R., Forastiere, F., Hakama, M., Heid, I., Kreienbrock, L., Kreuzer, M., Lagarde, F., Mäkeläinen, I., Muirhead, C., Oberaigner, W., Pershagen, G., Ruano-Ravina, A., Ruosteenoja, E., Schaffrath Rosario, A., Tirmarche, M., Tomášek, L., Whitley, E., Wichmann, H.-E., and Doll, R.: Radon in homes and risk of lung cancer: collaborative analysis of individual data from 13 European case-control studies, Brit. Med. J., 330, 223-226, 2005.

ICRP (International Commission on Radiological Protection), Publication 103: The 2007 Recommendations of the International Commission on Radiological Protection, Ann. ICRP, 37(2-4), 1332, 2007.

Papworth, D. S.: A need to reduce the radon gas hazard in the UK, J. R. Soc. Promo. Health, 117, 75-80, 1997.

Petford, N., Wertheim, D., and Miller, J. A.: Radon track imaging in CR-39 plastic detectors using confocal scanning laser microscopy, J. Microsc., 217, 179-183, 2005.

Phillips, P. S., Denman, A. R., Crockett, R. G. M., Gillmore, G., Groves-Kirkby, C. J., and Woolridge, A.: Comparative Analysis of Weekly vs. Three monthly radon measurements in dwellings, DEFRA Report No. DEFRA/RAS/03.006, 2004.

Wertheim, D., Gillmore, G., Brown, L., and Petford, N.: A new method of imaging particle tracks in Solid State Nuclear Track Detectors, J. Microsc., 237, 1-6, 2010. 